\title{
Visualizing Rayleigh Scattering through UV Photography
}

By ANDERs V. Lindfors ANd LASSE YLIANTTILA

BACKGROUND. Scattering of sunlight by air molecules is commonly known as Rayleigh scattering, and its scattering efficiency $\left(Q_{s}\right)$ is (approximately) inversely proportional to the fourth power of the wavelength $(\lambda)$ : $Q_{s} \propto \lambda^{-4}$. Thus, the shorter the wavelength, the stronger is the scattering. The blue color of the cloud-free sky is a well-known consequence of this relationship, while it is also known that radiation in the ultraviolet (UV) is even more diffuse than blue light.

These well-known facts are illustrated in Fig. 1, showing the Rayleigh optical depth as a function of wavelength together with the fractional contribution of the diffuse radiation to the global horizontal irradiance (diffuse fraction) under cloud-free conditions. The figure constitutes a typical textbook example of how this subject may be presented. It illustrates, first, the striking, almost5-fold differencebetween the Rayleigh optical depth at UV (365 nm) and midvisible (530 nm) wavelengths. Second, it shows the diffuse fraction as a function of solar zenith angle (SZA) for 4 different wavelengths, from $365 \mathrm{~nm}$ in the UV to $590 \mathrm{~nm}$ on the red side of the visible. The wavelengths shown in the figure correspond to the response of the channels of the camera that we have used for taking the photographs presented further on in this article.

The wavelength dependence of the diffuse fraction follows from the wavelength AFFILIATIONS: LINDFORS-Finnish Meteorological Institute, Kuopio, Finland; YLIANTTLLA-Radiation and Nuclear Safety Authority, Helsinki, Finland CORRESPONDING AUTHOR: Anders V. Lindfors, Finnish Meteorological Institute, Atmospheric Res. Center of Eastern Finland, P.O. Box 1627, (Yliopistonranta IF), Kuopio, 702II, Finland E-mail: anders.lindfors@fmi.fi

DOI:I0.II75/BAMS-D-I4-00260.I

A supplement to this article is available online (10.1175/BAMS-D-14-00260.2)

C2016 American Meteorological Society dependence of the Rayleigh optical depth, with stronger scattering and therefore higher diffuse fraction at shorter wavelengths. At a SZA of $60^{\circ}$, for example, the diffuse fraction at $365 \mathrm{~nm}$ is close to 0.5 , which is more than 4 times as much as the diffuse fraction at $530 \mathrm{~nm}$. The dependence on SZA, in turn, has to do with the increase of the optical path of the direct beam with lower sun (larger SZA); with the sun in the horizon $\left(\mathrm{SZA}=90^{\circ}\right)$, only diffuse radiation can reach a horizontal surface. In other words, this means that the diffuse fraction equals 1 for all 
wavelengths with the sun in the horizon. Note, however, that so strong is the Rayleigh scattering at $365 \mathrm{~nm}$, that the diffuse fraction virtually reaches 1 already at a SZA of $\geq 85^{\circ}$.

The skin-damaging radiation at wavelengths below $320 \mathrm{~nm}$ is even more diffuse (not shown in the figure) than that at $365 \mathrm{~nm}$. To illuminate a potential effect of the high diffuse fraction of the skin-damaging radiation under a moderately high Sun (SZA approximately $40^{\circ}-$ $60^{\circ}$ ), one sometimes hears mention of the fact that it is possible to get a sunburn (erythema) even when not exposed to direct sunlight but rather only to the diffuse skylight.

Although the paragraphs above aim at explaining the

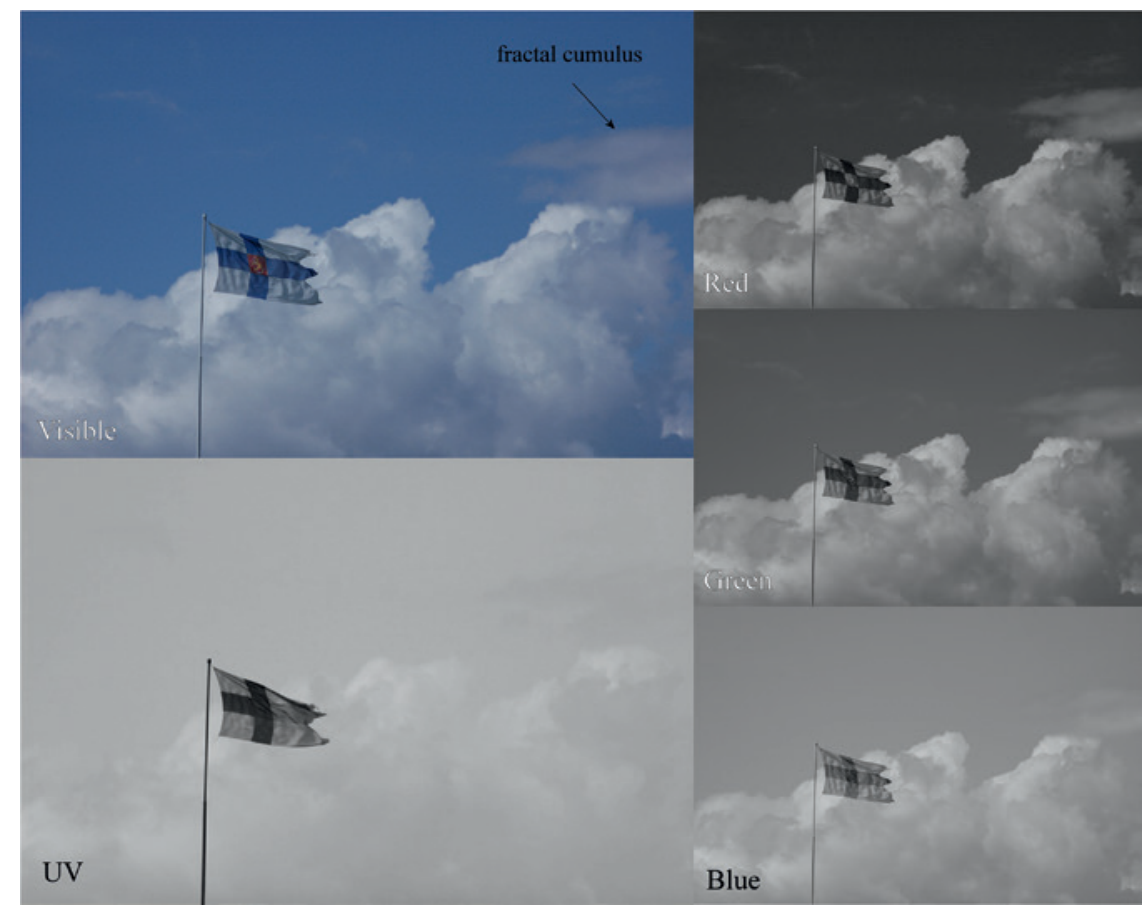

Fig. 2. A sequence of photographs of a blue sky, a flag (white and blue), and a cumulus cloud. The photographs represent different wavelength bands (clockwise starting from upper left: combined visible, red, green, blue, and $U V)$. This sequence demonstrates the increasingly diffuse sky radiance toward shorter wavelengths. Helsinki, $30 \mathrm{Jul} 2009$, III 10 UTC, SZA $=42^{\circ}$. basics of Rayleigh scattering and its consequences in terms of the wavelengthdependent distribution of diffuse radiation, we find that this is a subject that is not always easy to understand in a concrete way. Below, we illustrate the physics of the Rayleigh scattering atmosphere in a more intuitive way, using photographs representative of radiation in the UV band together with photographs using the standard red, green, and blue channels. Our aim is to connect the typically abstract and theoretical information of textbooks with a more visually intuitive representation of the effects of Rayleigh scattering.

UV PHOTOGRAPHS. A tailored camera setup was used to take photographs of the atmosphere and the environment as seen in the UV band. The camera was an unmodified Nikon D70s digital camera, which happens to be sensitive to UV radiation, especially in its red channel. Considering that the camera is intended to record visible light, this is a fault. From the perspective of UV photography, on the other hand, this is fortunate because it makes it possible to use this camera to take photographs representative of UV radiation.

In addition to UV sensitivity of the camera, UV photography requires that the lens transmits UV radiation. In addition, the unwanted part of the spectrum needs to be filtered out. Here, a Nikon UV Nikkor $105 \mathrm{~mm}$ objective was used, which is a purpose-built lens for UV, visible, and infrared photography. The lens has the same focal length for UV and visible light, thus making UV photography easier as the there is no need for focus adjustment between UV and visible light photographs. For photographs in the visible channels, a UV-blocking filter was used, while for the UV photographs, a 11/4" Baader U-Filter-blocking visible light was used. For the UV photographs, the original image was split into its red, green, and blue components, and the red channel image was used. Finally, the photograph was converted to a black-and-white image.

The final UV photograph corresponds to the wavelength range from $350 \mathrm{~nm}$ to $390 \mathrm{~nm}$, with peak response at approximately $365 \mathrm{~nm}$. The red, green, and blue channels of the camera have their peak response at $590 \mathrm{~nm}, 530 \mathrm{~nm}$, and $460 \mathrm{~nm}$, respectively, as indicated also in Fig. 1. 


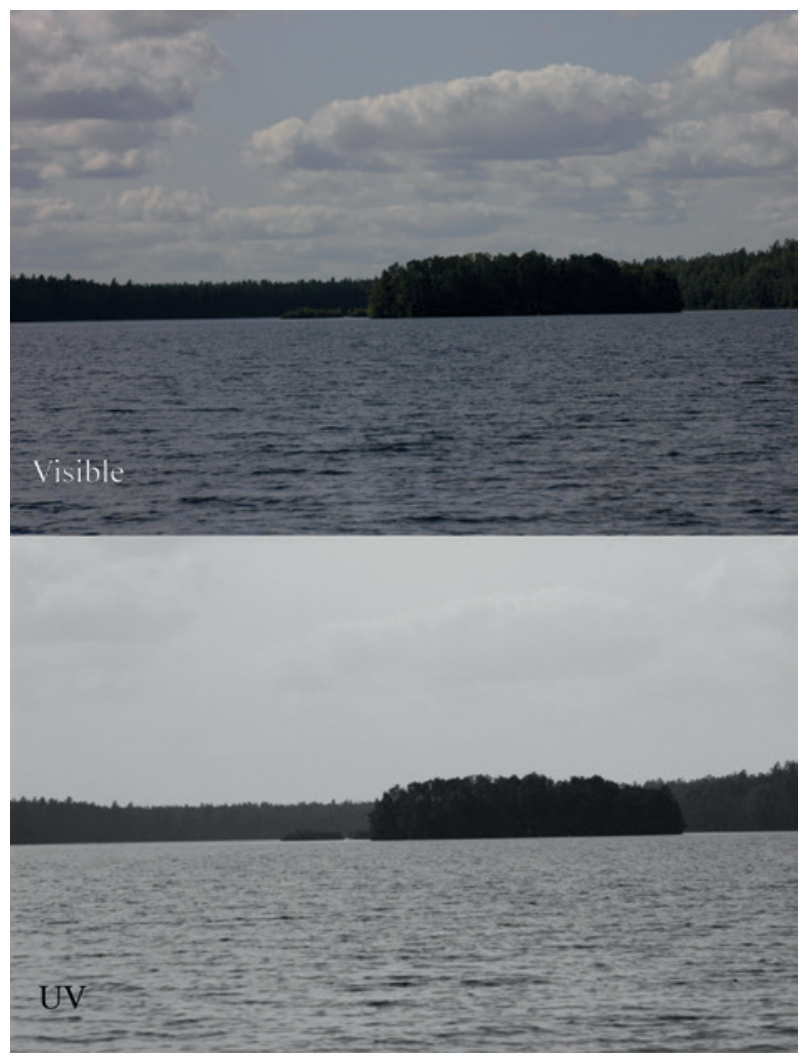

Fig. 3. A Finnish lake landscape under cumulus clouds. The photographs are representative of the (top) combined visible and (bottom) UV channel. Hyvinkää, 2 I Aug 2013, 1000 UTC, SZA $=49^{\circ}$.

Figure 2 shows a sequence of photographs of the same scene, taken with only a short time difference $(<1 \mathrm{~min})$ in between, so that the conditions such as SZA and cloud optical properties can be assumed to be essentially the same in all photographs. The scene consists of a blue sky, a Finnish flag (white and blue), and a cumulus cloud (white). This sequence of photographs makes visible what the human eye cannot normally perceive-in particular, the effects of the increasingly strong scattering of radiation of shorter wavelengths by the molecular atmosphere. In the red, there is a stark contrast between the white cumulus cloud and the dark sky. The sky appears increasingly bright toward shorter wavelengths. In the UV band, the cloud almost disappears into the strong diffuse background radiation of the sky.

There are also some fractal cumulus clouds present in Fig. 2, with a particularly distinct one on the righthand side of the image just above the main cumulus cloud. It is worth noting how the appearance of this fractal cumulus cloud varies between the different

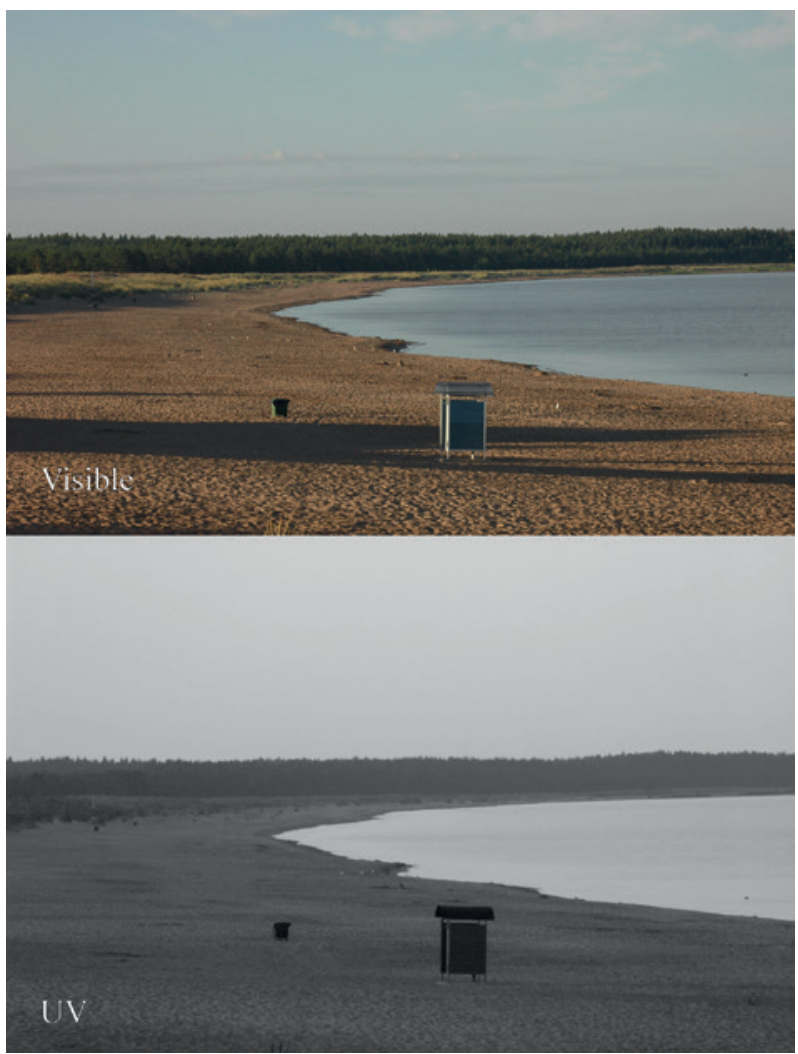

FIG. 4. Photographs taken early in the morning demonstrate the high diffuse fraction of $U V$ radiation as compared to visible. (top) There are strong shadows in the visible photograph, whereas (bottom) the shadows essentially disappear in the UV photograph. Pori, 18 Jul 20I4, 0314 UTC, SZA = $81^{\circ}$.

channels. In the combined visible, red, green, and blue channel, this cloud is visible as an area lighter than the sky, while in the UV, it appears somewhat darker than the sky. This indicates that the optical depth of this cloud is small enough so that the cloud reflects only weakly, but still large enough for the cloud to be brighter than the background sky in the visible channels. In the UV, it seems plausible that the effect of the cloud blocking the diffuse sky radiance dominates, and it therefore appears darker than the background. In practice, this would indicate that the optical depth of this cloud is of the same order of magnitude as that of Rayleigh scattering in the UV.

Figure 3 provides another striking example of how cumulus clouds, making the sky heterogeneous in the visible, become hard to distinguish in the UV photograph. The figure also demonstrates how dark objects (the trees) appear lighter with increasing distance. This aerial perspective is seen more clearly 
in the UV than in the visible because of the stronger scattering in the UV.

When the sun is low (large SZA), the portion of scattered diffuse light is high, in particular in the short wavelengths. As discussed above, essentially all of the radiation in the UV band can be diffuse under these conditions (see also Fig. 1). This is further illustrated in Fig. 4. The photograph was taken early in the morning with a SZA of around $80^{\circ}$. There are stark shadows in the visible photograph, while in the UV, there are practically no shadows.

DISCUSSION. Using UV photography, this article illustrates the effect of the strongly wavelengthdependent Rayleigh scattering by air molecules. The photographs presented here make visible what human beings cannot normally see. We have found these photographs to be eye-opening, and one aim of this article is to share this experience with the scientific community.

Only a small subset of the photographs taken is presented here. Some additional photographs are made available as an electronic supplement to this article, including high-quality versions of the photographs included in the main article (http:// dx.doi.org/ | 0.I I75/BAMS-D-| 4-00260.2).

Finally, it is worth noting that phenomena other than Rayleigh scattering can also be studied and demonstrated using UV photography. These phenomena include, for example, the reflection of UV radiation by water surfaces, which to some extent is also present in both Fig. 3 and Fig. 4.

ACKNOWLEDGMENTS. AVL was supported by the Academy of Finland, decision 284536.

\section{FOR FURTHER READING}

Bohren, C. F., and A. B. Fraser, 1985: Colors of the sky. Phys. Teach., 23, 267-272, doi:10.1119/1.2341808.

Lilienfeld, P., 2004: A Blue sky history. Opt. Photonics News, 15, 32-39, doi:10.1364/OPN.15.6.000032.

Mayer, B. and A. Kylling, 2005: Technical note: The libRadtran software package for radiative transfer calculations-description and examples of use. Atmos. Chem. Phys., 5, 1855-1877, doi:1680-7324 /acp/2005-5-1855.

Rørslett, B., cited 2015: All you ever wanted to know about digital UV and IR photography, but could not afford to ask. [Available online at www.naturfotograf .com/UV_IR_rev00.html.]

World Health Organization (WHO), posted 2002: Global solar UV Index: A practical guide. [Available online at www.who.int/uv/publications/en/UVIGuide.pdf.] Young, A. R., L. O. Björn, J. Moan, and W. Nultsch, 1993: Environmental UV Photobiology. Plenum Press, 479 pp. Young, A. T., 1981: Rayleigh scattering. Appl. Opt., 20, 533-535, doi:10.1364/AO.20.000533. 